\title{
Study on Intelligent Stowage of Container Ship
}

\author{
Mingjie Li \\ Marine college of Shandong jiaotong university, Weihai, Shandong,China
}

Keywords: Container ship, intelligent stowage, combinatorial optimization, constraint bin packing problem

\begin{abstract}
Operation of cargo ship transport in the production of a very important link. Stowage of containers directly related to safety and efficiency of maritime transport, how many feasible options in seeking a relatively optimal stowage plan to ensure ship safety, reduce transport costs, improve operational efficiency and competitiveness, is the shipping industry one of the main objectives pursued. In a large number of documents on the basis of focusing on the method of container ships ' intelligent stowage, finally, an example is developed on the prototype of a container ship's stowage system was verified.
\end{abstract}

\section{Introduction}

International container transportation by sea is 60 an modern mode of transport in the world, it has incomparable advantages of general cargo transportation. To shorten transit time of goods, save costs, reduce damage and cargo, speed up shipping turnover, especially easier to organize door-to-door and international multimodal transport. Have great significance for the promotion of international trade. With the deepening of reform and opening up and development of China's maritime container transport is greatly.

Container ship cargo is containerized maritime transport is an important step to ensure ship safety, cargo safety and guarantee schedule has an important role. Traditional methods of loading is determined by Chief Officer or Terminal experience done by hand. Now, due to the rapid development of container transport at sea and expansion of container tonnage, manual loading is very difficult to do it. The advent of the computer, for the automatic loading of containers makes it possible, as early as the beginning of container transportation development in the 60 's, abroad, some shipping companies and research units to many useful explorations, and achieved some results. Evidently loading method of new research, try to develop container shipping intelligence stowage system was necessary, but also has important theoretical and practical significance.

Container transport started late in China, Chongqing and ship refit over the small ship, tonnage is small, so much later than the foreign container stowage research. Until the late 80 's before starting a number of computer models.

Back in the late 60 and early 70 's, foreign shipping companies and research institutions on container ship's stowage methods had a strong interest in research, the objective of the study is to find a meet the established conditions, pour boxes at least, high efficiency of the loading plan.

\section{Container vessel stowage}

Container is a certain strength, stiffness and specifications specifically for working capital using large shipping containers, transport bales or without loading the package into a set of tools (container) in General.

Container specifications according to international standards, area standard, national standards and company standard and different. international standards container is by ISO developed of standard and manufacturing of container. currently international shipping container in the usually are used $20 \mathrm{ft}$ and $40 \mathrm{ft}$ long of two species container. for makes container box number calculation unified of, put 20ft container as a calculation units, with TEU said, $40 \mathrm{ft}$ container as two a calculation units, to unified calculation container of operating volume. 
Container vessel stowage result is represented in the figure, the Bay plan, practical, and final stowage plan, practical and final stowage plan is based on the Bay plan, its preparation process in the following programs:

Distribution: by the shipping company, container loading center according to the ship's voyage booking, preparation of the ship's voyage at the port of loading container Bay plan, and this figure for container handling company directly or by fax, telex, sent by way of shipping agents, then transferred to the container handling company.

Practical: the dock master or container stowage of container handling company, according to the Bay plan and terminal boxes stacked, without violating the Bay plan proposes stowage under the principle of preparing practical container.

Review: after the container ship berthing, dock Planner practical boat review referred to the Chief Officer, ships, agree to sign, the stevedoring company in real assignment, to container ship loading.

Stowage: container shipping is completed, by the tallymen as actual packing, preparing final stowage plan.

\section{Discussion on method of container ships ' intelligent stowage}

Containership loading problem is with complex constrained multiobjective combinatorial optimization problems, due to ship loading strength, stability, more territory, characteristics and fast loading and unloading of the container, making to solve the problem very complex.

Effect stowage of main factors antibody up can is divided into two a aspects, a is ship security factors, main including full using container ship hosted capacity, guarantee ship has moderate stability sex, appropriate of strength and draft poor; another aspects is effect transport economic sex of factors, main including how in full of State Xia maximum to using ship of Mount capacity, towards more boxing, meet different purpose Hong Kong container Hong Kong sequence of requirements reduced Midway of pour box and the handling convenient and improve handling efficiency, aspects of requirements, also, Also the container size, type of goods, container ship structures and so on. So when the container ship's stowage, constraints, objectives, and they subsequently bound to each other, interrelated, solve difficult.

From literature to establish mathematical model of loading, is a nonlinear integer programming problems, and integer programming problem is the NPC problem. Has proof container loading problem is NP-hard combinatorial optimization problems with complex constraints, the scale of the problem and ship container capacity, anchored in each port port number and container number, especially when shipping container capacity, call port for a long time, cargo also increased rapidly. Even for a small quantity of loading issues due to too many factors to be considered are large and complex optimization problems.

Using modern optimal algorithm of heuristic algorithms (genetic algorithm and tabu search algorithm, simulated annealing, etc) looking for better loading problem possible solution structures correspond to the problem solving algorithm, which can greatly reduce the space of feasible solutions, we provide a way to solve complex.

Combinatorial Optimization (combinatorial optimization) is through the study of mathematical methods to find the optimal arrangement of discrete events, groups, order or screening. is a typical and important branch of research questions related to information technology, project management, transportation and communications networks and many other areas. as shown in Figure 1. 


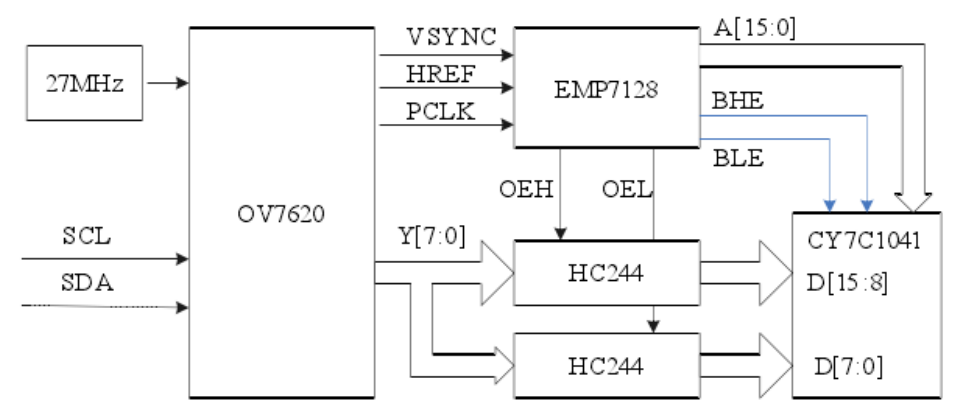

Fig . 1 Combinatorial optimization

Stowage Shi, due to a purpose Hong Kong of container number may is greater than ship Shang Bay bit in the box bit number, so to consider items size over maximum box capacity Shi of mount problem, at must split points super size items to boxing, in qualified split points times of conditions Xia items can by any proportion split points, how will all items loaded into several box in the, makes by with box bit of total capacity minimum, called super; size items boxing problem. pre distribution Shi according to super size items boxing algorithm solution.

Algorithm steps are as follows:

Step one, called "staining boxing" heuristic;

Step two, initialize the cargo space with packing matrix;

(1) initial load space, that is arranged by Bay, from the bow to the stern, assigning values to each Bay (fill up the empty containers);

(2) initial boxed matrix;

Stage three, the first port container loading;

(1) containers to the port of destination is divided into subsets of $p$ similar box set, $p=\{1,2,,, p\}, p$ for the number of ships link ports;

(2) the sort of diminishing this subset p according to the port of destination, that is, as far as the port of destination box set in the first, long came in second, followed by analogy; the destination box sets of the same kind as far as was the first to be assembled, much as a second, repeat;

(3) packing algorithm is called, the current load of containers in ports; if a port's container volumes greater than any Bay the number of empty slots, then the destination container is split into two parts, according to two articles to load until the split until the remaining can be loaded with a box;

(4) check the vertical constraints are met, does not satisfy criteria, swaps the same weight of box section in the Bay, until the constraint condition is met;

Steps four, for non-first a mount Hong Kong, to uninstall Hou of box bit State for current Hong Kong of initial state, update box capacity and the number, also will similar container group by purpose Hong Kong decline sort, calls boxing algorithm, as evaluation function for mount. If not for zero, is said current Bay bit not for mount this purpose Hong Kong of container, again again looking for other of Bay bit, makes value minimum and meet constraints conditions requirements of location is optimal mount location;

Step five, all the current completion of the loading of containers in ports, go five steps else go step four;

Step six, the output load results, ended.

To pour box number at least, ship contains box rate highest and the different purpose Hong Kong container occupied Bay bit number at least for target, to meet ship longitudinal strength and draft poor requirements for constraints conditions, established pre distribution optimization model; with similar box group as processing unit, used based on II fork search tree of optimal match boxing algorithm on problem solution, will similar box group distribution to ship different Bay bit Shang, achieved has container in ship Shang of longitudinal configuration. 


\section{Design of container ship's stowage system}

Ship involved in this system include:

(L) the basic information such as the captain of the ship, breadth, moulded depth, load, displacement, container capacity

(2) the general arrangement plans for ships, ship shape charts, table of offsets;

(3) sheet of hydrostatic curves, stability cross curve table, Bonjean curve table angle curve the water table;

(4) on board a vessel at the specified rib allowable shear and the allowable bending moment values, empty weight curve, ribbed coordinates;

(5) each line box bitmap, each box in the ship's coordinates and center of gravity.

This system is mainly used visualBasic6.0 as the development platform of Visual, design the main interface for the programs and features module in order MS-Access as a back-end database (such as storing a variety of numerical data in the form of a table), use ADO for database access technology program associated data in a table. In order to calculate the convenience and avoid repetitive work, introduces the MatrixVB component for processing. Through its interface with VB programming using Matlab functions provided in the MatrixVB library to calculate the processing of data.

The main interface of the system, including slotting operations window, liquid tanks enter Windows, basic calculation window, check window of stability and strength check of Windows. the main window as shown in Figure 3:

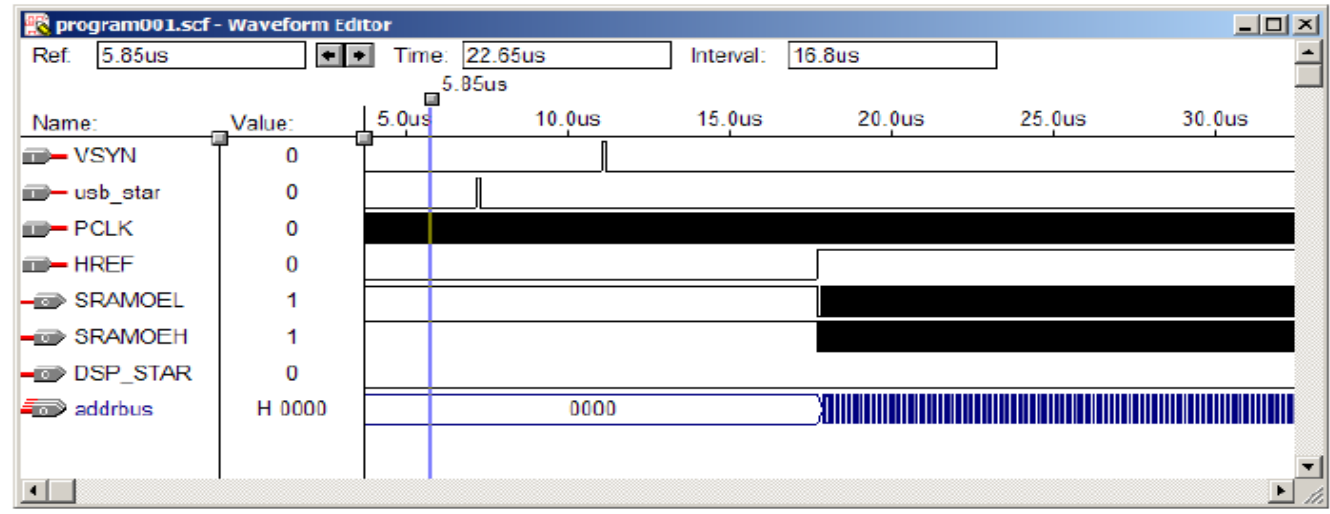

Fig . 2 Main interface of the System

Depending on the stowage plan a relevant evaluation model, and compare the advantages and disadvantages of different methods for solving problems with a view to finding a more suitable method of loading problem. for a container ship's stowage system, due to the rapid development of network technology and artificial intelligence technology, so you can consider design based on Internet system and Agent technology to realize the remote loading of the goods, and develop truly intelligent loading system.

\section{References}

[1]Qiu Wenchang Qiu Qiang. a calculation method for ship trim. marine technology, 2003 (2)

[2]Sun Guoyuan Moltke Huang. study on automatic detection of ship's draught and stability parameters. sailing in China, 2011

[3]Xu Bangzhen Jiang Weiqing. optimization of container ship heavy distribution. Journal of Dalian Maritime University, 2012 (3)

[4]J.Jesus, S.Hannafi,F.Semet.The Container Assignment Problem: Models and Solution Methods. Odysseus 2003-Seientific Program

[5]Joaquin Garcia, Gilberto Viveros, Rene Cumplido. FPGA based Architecture for Radar's STC, FTC and Gain modules. México: INAOE, 2002 
[6]David K. Barton, Sergey A. Leonov. Radar Technology Encyclopedia. Artech House, 2008 [7]Texas Instruments . TMS320DM642 Fixed-Point Digital Signal Processor Data Manual. www.ti.com, 2005

[8] Peng Qizong, Li Yubai, Guan Qing. Development and application of DSP technology. Higher education press, 2009 